\title{
Atividade antimicrobiana do biossurfactante produzido por Pseudomonas sp. CCMICS 105
}

Antimicrobial activity of biosurfactant produced by Pseudomonas sp. CCMICS 105

\author{
T. S. Oliveira; S. C. Santos*; S. C. Silva; A. C. V. Campos \\ Laboratório Multiuso de Biologia / Laboratório de Química / Laboratório e Observatório em Vigilância e \\ Epidemiologia Socia, Universidade Federal do Sul e Sudeste do Pará (Unifesspa), 68507-590, Marabá-Pará, Brasil \\ *sidnei.cerqueiradossantos@gmail.com \\ (Recebido em 07 de setembro de 2021; aceito em 13 de janeiro de 2022)
}

\begin{abstract}
O uso irracional e indevido de antibióticos tem proporcionado condições favoráveis para desencadear a resistência dos microrganismos. Os biossurfactantes têm apresentado propriedades farmacêuticas promissoras, principalmente relacionada com a função antimicrobiana. Nesse sentido, o objetivo desse trabalho foi avaliar a atividade antimicrobiana do biossurfactante produzido pela linhagem Pseudomonas sp. CCMICS 105 contra cepas microbianas patogênicas. A produção do biossurfactante foi realizada em meio salino mineral e o tensoativo foi extraído com a mistura dos solventes clorofórmio e etanol. A atividade antimicrobiana foi avaliada contra as cepas Escherichia coli, Staphylococcus aureus, Bacillus subtilis e Candida albicans, usando os testes de concentração inibitória mínima (CIM) e concentração bactericida mínima (CBM). O biossurfactante produzido pela linhagem bacteriana inibiu todas as cepas testadas, apresentado resultados equivalentes para CIM e CBM. A prospecção de biossurfactante com potencial antimicrobiano é uma alternativa para descobertas de novos antibióticos.

Palavras-chave: antibiótico, ramnolipídio, microrganismos patogênicos.
\end{abstract}

The irrational and inappropriate use of antibiotics has provided favorable conditions to trigger the resistance of microorganisms. Biosurfactants have shown promising pharmaceutical properties, mainly related to their antimicrobial function. In this sense, the aim of this work was to evaluate the antimicrobial activity of biosurfactant produced by Pseudomonas sp. CCMICS 105 against pathogenic microbial strains. The production of the biosurfactant was carried out in a mineral saline medium and the surfactant was extracted with a mixture of chloroform and ethanol solvents. The antimicrobial activity was evaluated against Escherichia coli, Staphylococcus aureus, Bacillus subtilis and Candida albicans strains, using the minimal inhibitory concentration (MIC) and minimal bactericidal concentration (MBC) tests. The biosurfactant produced by the bacterial strain inhibited all strains tested, showing equivalent results for MIC and MBC. The prospect of biosurfactant with antimicrobial potential is an alternative for discovering new antibiotics.

Keywords: antibiotic, rhamnolipid, pathogenic microorganisms.

\section{INTRODUÇÃO}

Os biossurfactantes são moléculas anfipáticas de superfície ativa produzidos por microrganismos na superfície celular ou secretados extracelularmente [1]. Estas moléculas possuem as propriedades de reduzir as tensões superficial nas interfaces ar-água e interfacial nas interfaces óleo-água, atividade emulsificante, estabilidade em condições extremas de $\mathrm{pH}$, temperatura e salinidade, baixa toxidade, biodegradabilidade [2, 3], além de poder ser produzidos a partir de fontes renováveis [4-6].

Os biossurfactantes são classificados em diferentes grupos, como glicolipídios, lipopeptídios, fosfolipídios, compostos poliméricos e lipídios neutros. Esta classificação é feita conforme a composição química e origem microbiana, podendo ser influenciada pela natureza do substrato, concentrações de íons no meio de cultura e condições de cultivo. [7, 8].

Os surfactantes microbianos têm sido amplamente aplicados na recuperação avançada de petróleo, biorremediação e emulsificação industrial $[4,9,10]$, mas também há relatos que os biossurfactantes possuem propriedades de importância terapêutica e biomédica, como as atividades antibacteriana e antifúngica $[11,12]$. 
Os biossurfactantes que têm apresentado atividade antimicrobiana são surfactina e iturina produzidas por Bacillus subtilis [13], soforolipídios produzidos por Candida bombicola [14], lipídios de manosileritritol por Candida antarctica [15], ramnolipídios por Pseudomonas aeruginosa [16].

Os estudos da propriedade antimicrobiana dos biossurfactantes têm apresentado resultados promissores, representado uma alternativa estratégica para descoberta de novos agentes antimicrobianos. Nesse contexto, o objetivo desse trabalho foi avaliar a atividade antimicrobiana do biossurfactante produzido pela linhagem Pseudomonas sp. CCMICS 105 contra cepas microbianas patogênicas.

\section{MATERIAL E MÉTODOS}

\subsection{Microrganismos}

O biossurfactante utilizado foi produzido pela linhagem Pseudomonas sp. CCMICS 105 (GenBank n ${ }^{\circ}$ MW381109.1), conhecida inicialmente como Pseudomonas fluorescens Slim15 $[5,17,18]$. Para o teste de atividade antimicrobiana foram selecionadas as cepas oriundas da American Type Culture Collection (ATCC): Candida albicans (ATCC 40175), Escherichia coli (ATCC 8739), Staphylococcus aureus (ATCC 25923) e Bacillus subtilis (ATCC 23857). Os microrganismos foram conservados em meio Hogness a $-20^{\circ} \mathrm{C}$ no Laboratório Multiuso de Biologia da Universidade Federal do Sul e Sudeste do Pará (Unifesspa).

\subsection{Meio de cultivo e condições de crescimento}

O meio salino mineral (MSM) foi utilizado no ensaio de produção de biossurfactante, com a seguinte composição (g/L): $\mathrm{K}_{2} \mathrm{HPO}_{4}, 4,0 \mathrm{~g} ; \mathrm{Na}_{2} \mathrm{HPO}_{4}, 1,5 \mathrm{~g} ; \mathrm{NaNO}_{3}, 1,0 \mathrm{~g} ; \mathrm{MgSO}_{4} .7 \mathrm{H}_{2} \mathrm{O}, 0,2 \mathrm{~g}$; $\mathrm{CaCl}_{2} \cdot 2 \mathrm{H}_{2} \mathrm{O}, 0,02 \mathrm{~g} ; \mathrm{FeCl}_{3} \cdot 6 \mathrm{H}_{2} \mathrm{O}, 0,02 \mathrm{~g}$ [19], com 0,5\% de glicerol como substrato, sendo ajustado o $\mathrm{pH}$ para $7.0(\mathrm{HCl}$ ou $\mathrm{NaOH})$. Erlenmeyer $(125 \mathrm{~mL})$ contendo MSM $(30 \mathrm{~mL})$ foi esterilizado $\left(121^{\circ} \mathrm{C}\right)$ por 20 minutos. Foi inoculado $2,5 \mathrm{~mL}$ da suspensão bacteriana, ajustado a partir da escala 0,5 de McFarland. O controle negativo foi MSM não inoculado. O Erlenmeyer foi incubado no agitador orbital a $180 \mathrm{rpm}\left(30^{\circ} \mathrm{C}\right)$ por 48 horas. Em seguida, a cultura foi centrifugada (4000 rpm) por 20 minutos [16]. A presença de biossurfactante no caldo livre de céluas foi confirmada através da atividade emulsificante.

$\mathrm{O}$ índice de emulsificação $\left(\mathrm{IE}_{24}\right)$ foi medido usando a seguinte fórmula: $\mathrm{IE}_{24}(\%)=\mathrm{x} / \mathrm{y} \times 100$, onde $\mathrm{x}$ representa a altura da camada emulsificada $(\mathrm{mm})$ e y a altura total (mm) [20]. O MSM não inoculado e sodium dodecyl sulfate (SDS) a $1 \%$ foram utilizados como controles.

\subsection{Extração do biossurfactante}

A extração do biossurfactante extracelular foi realizada de acordo com Lotfabad et al. (2009) [21], misturando $180 \mathrm{~mL}$ do caldo livre de células com $360 \mathrm{~mL}$ da mistura dos solventes clorofórmio e etanol $(2: 1, \mathrm{v} / \mathrm{v})$. A solução foi agitada e mantida em repouso por 24 horas em um balão de separação, até separação das fases. Este procedimento foi realizado por três vezes. A fase orgânica foi evaporada em evaporador rotativo a $40^{\circ} \mathrm{C}$ para obtenção do biossurfactante bruto e ressuspensa em $5 \mathrm{ml}$ de água ultrapura (solução mãe).

\subsection{Determinação da atividade antimicrobiana}

A atividade antimicrobiana do biossurfactante bruto foi testada contra as cepas ATCC, usando o método da concentração inibitória mínima (CIM), de acordo com as recomendações da National Committee for Clinical Laboratory Standards (2003) [22]. O ensaio de microdiluição 
em caldo foi realizado em microplaca de 96 poços, sendo adicionados $90 \mu \mathrm{L}$ do caldo MuellerHinton $(\mathrm{CMH})$ (Kasvi) nos poços e transferido $100 \mu \mathrm{L}$ da solução de biossurfactante bruto $(80 \mathrm{mg} / \mathrm{mL})$, por meio da técnica de diluição seriada. Após essa etapa, foi aplicado $10 \mu \mathrm{L}$ da suspensão bacteriana. A solução de clorofórmio e etanol (2:1, v/v) e o antibiótico tetraciclina $(30 \mu \mathrm{g} / \mathrm{disco})$, para as bactérias, foram utilizados como controle. A placa foi incubada a $37^{\circ} \mathrm{C}$ por $24 \mathrm{~h}$ para as bactérias e $48 \mathrm{~h}$ para levedura. A solução reveladora cloreto de 2,3,5 trifeniltetrazólio (TTC) foi aplicada nos poços para avaliar o crescimento microbiano, e incubada na estufa por mais 2 horas, sendo considerando crescimento microbiano a presença de coloração rosa avermelhada e inibição do crescimento a ausência de coloração [23].

A concentração bactericida mínima $(\mathrm{CBM})$ foi realizada levando em consideração os resultados preliminares no ensaio de CIM. Foi transferido uma alçada da cultura dos poços que apresentaram inibição no teste de CIM, com o auxílio da alça bacteriológica, e semeada por esgotamento na superfície do meio TSA. O crescimento das colônias bacteriana e leveduriforme foi avaliado após o período de 24 a 48 horas de incubação $\left(37^{\circ} \mathrm{C}\right)$.

Os testes foram realizados em triplicata. A análise descritiva dos dados foi realizada no programa Microsoft Excel versão 2010.

\section{RESULTADOS E DISCUSSÃO}

\subsection{Produção de biossurfactante}

Os biossurfactantes formam um grupo estruturalmente diverso de moléculas tensoativas sintetizadas por microrganismos [24]. Estes são geralmente isolados de diferentes ambientes, como solos e fontes de água contaminados com hidrocarbonetos [12, 25], rejeito de áreas de exploração de minério, rizosfera de espécies vegetais. A linhagem Pseudomonas sp. CCMICS 105 foi isolada no Parque Municipal de Mucugê, região semiárida do estado da Bahia, a partir do solo rizosférico e adjacente de Actinocephalus spp., pertencente à família Eriocaulaceae [17].

O gênero Pseudomonas é amplamente conhecido quanto a capacidade de produzir biossurfactante, principalmente o pertencente a classe glicolipídio. O glicolipídio constitui um dos mais amplos grupos de biossurfactantes estudados, sendo que o composto mais conhecido é o ramnolipídio, produzido principalmente pela espécie $P$. aeruginosa $[10,16,26]$. Entretanto, também há relatadas de outras espécies de Pseudomonas produtoras de ramnolipídios, como $P$. chlororaphis, $P$. plantarii, $P$. putida e $P$. fluorescens [27]. A análise do gene sequenciado $16 \mathrm{~S}$ rRNA da linhagem bacteriana CCMICS 105 indicou que há 99,93\% de similaridade com a espécie Pseudomonas aeruginosa (Dados não mostrados).

O ramnolipídio é composto por moléculas de ramnose ligadas a uma, duas ou três cadeias de ácido B-hidroxidecanóico, de comprimento variado de 8 a 22 carbonos [28, 29]. O teste de orcional revelou que possivelmente o biossurfactante produzido pela linhagem Pseudomonas sp. CCMICS 105 é do tipo ramnolipídio, devido a presença de L-ramnose (190 mg/L) na composição da molécula [17].

O índice de emulsificação é uma técnica muito utilizada para determina a presença de biossurfactante no caldo livre de células [30]. Araújo et al. (2020) [16] mostraram que o ramnolipídio produzido pela linhagem Pseudomonas aeruginosa AP029-GLVIIA formou emulsificado usando diferentes fontes de carbono, variando de 58\% a 77\%. Das et al. (2014) [31] também relataram que o ramnolipídio produzido por Pseudomonas sp. foi capaz de formar índice de emulsificação entre 50\% a 80\%. A linhagem Pseudomonas sp. CCMICS 105 foi capaz de produzir biossurfactante em diferentes condições de cultivo (e.g. pH, temperatura, fontes renováveis de carbono), apresentando atividade emulsificante de até $63 \%$, e reduzindo as tensões superficial da água para $30 \mathrm{mN} / \mathrm{m}$ e interfacial do óleo cru para 2,41 mN/m [5, 17, 18]. Neste trabalho, o caldo livre de células da linhagem Pseudomonas sp. CCMICS 105 produziu índice de emulsificação médio de 62\%, após 48 horas de incubação (Figura 1), indicando que os biossurfactantes produzidos por espécies de Pseudomonas são potenciais agentes emulsificantes. O SDS apresentou $\mathrm{IE}_{24}$ de $65 \%$ e o MSM não inoculado não produziu emulsificado (Figura 1). 


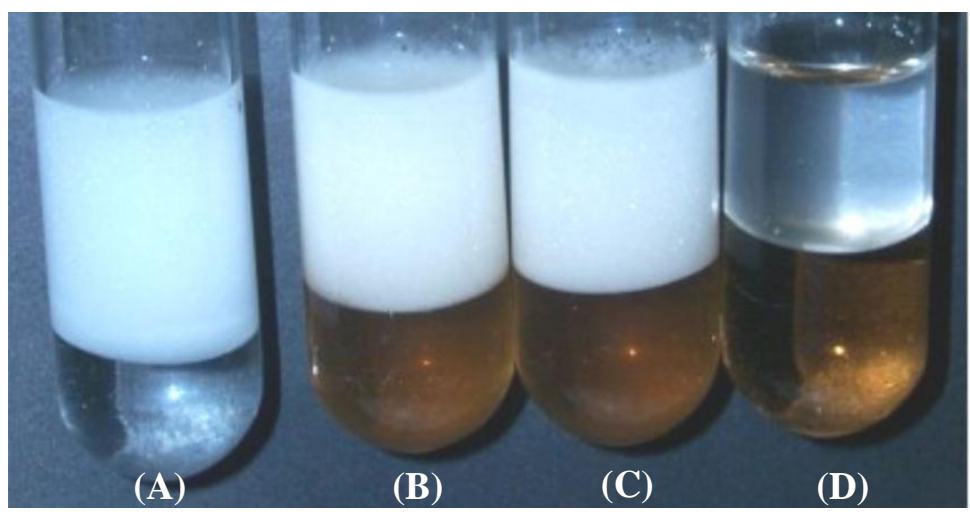

Figura 1: Produção de emulsificado pela linhagem Pseudomonas sp. CCMICS 105: A) Sodium dodecyl sulfate a 1\%; B e C) Caldo livre de células; e D) MSM não inoculado.

Um total de $800 \mathrm{mg}$ de massa seca de biossurfactante bruto foi extraído com os solventes clorofórmio e etanol, a partir de $180 \mathrm{~mL}$ de caldo livre de células da linhagem Pseudomonas sp. CCMICS 105. Após a ressuspensão, foi preparada uma solução de trabalho de $160 \mathrm{mg} / \mathrm{mL}$ para realização dos testes de atividade antimicrobiana.

As novas legislações de controle ambiental e a busca de produtos industriais menos impactantes têm levado a procura por surfactantes biológicos como alternativa aos surfactantes sintéticos, devido às propriedades físico-químicas e biológicas [10, 32-34], e a possibilidade de serem produzidos a partir de fontes renováveis $[4,5,6]$. Dentre os surfactantes biológicos, os ramnolipídios têm sido o mais utilizados para o desenvolvimento de processos de biorremediação [10] e pelas indústrias, destacando-se como um dos principais surfactantes renováveis [35].

\subsection{Atividade antimicrobiana}

A comunidade científica tem investigado ativamente produtos naturais com propriedade antimicrobiana, principalmente derivados de vegetais e de microrganismos [36-38]. Os surfactantes microbianos têm apresentado propriedades antimicrobianas de amplo espectro contra bactérias Gram-positivas, Gram-negativas e fungos [39, 40], tornando-se uma alternativa promissora aos antibióticos convencionais [41].

A atividade antimicrobiana foi determinada com base na concentração inibitória mínima. $\mathrm{O}$ biossurfactante produzido pela linhagem Pseudomonas sp. CCMICS 105 apresentou atividade antimicrobiana para todas as cepas microbianas testadas (Figura 2). As concentrações inibitória e bactericida mínimas das cepas ATCC foram equivalentes nos testes de CIM e CBM.

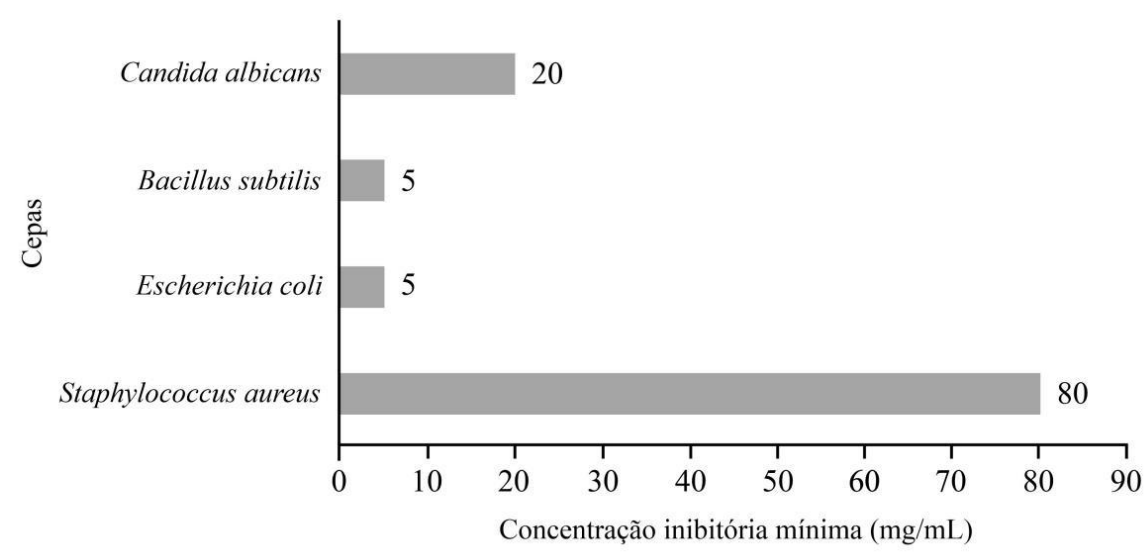

Figura 2: Concentração inibitória mínima do biossurfactante produzido pela linhagem Pseudomonas sp. CCMICS 105 contra cepas patogênicas. 
Estudos têm apresentado resultados promissores da atividade antimicrobiana dos ramnolipídios. El-Sheshtawy e Doheim (2014) [42] observaram que o ramnolipídio produzido por $P$. aeruginosa ATCC-10145 inibiu o crescimento das cepas Sarcina lutea, Bacillus pumilus e Candida albicans. Das et al. (2014) [31] relataram que o ramnolipídio produzido pelas linhagens de Pseudomonas apresentou atividade antimicrobiana contra E. coli, B. subtilis e $S$. aureus. Vijayakumar e Saravanan (2015) [43] demonstraram que o biossurfactante (glicolipídio) produzido pela linhagem $P$. aeruginosa $\mathrm{PB} 3 \mathrm{~A}$ foi capaz de controlar o crescimento de $E$. coli $\mathrm{e}$ $S$ aureus. Ndlovu et al. (2017) [11] relataram que o ramnolipídio produzido por $P$. aeruginosa ST5 inibiu o crescimento de E. coli e S. aureus. Mais recentemente, Araújo et al. (2020) [16] relataram o potencial antifúngico do ramnolipídio frente a espécies $C$. albicans e $C$. tropicalis. A linhagem Pseudomonas sp. CCMICS 105 foi capaz de inibir o crescimento tanto de bactérias (Gram-positivas e Gram-negativas) quanto do fungo leveduriforme, mas com graus diferentes de sensibilidade (Figura 2).

As bactérias Gram-negativas são normalmente mais resistentes à ação dos ramnolipíos do que as Gram-positivas, possivelmente devido ao lipopolissacarídeo (LPS) de membrana externa exclusivo das Gram-negativas, que pode atuar como barreira protetora entre a membrana interna e a parede celular contra compostos tóxicos [44]. Segundo El-Sheshtawy e Doheim (2014) [42], a molécula de ramnolipídio pode inibir a atividade bacteriana por meio da inserção dos componentes de ácido graxo na membrana celular, causando alterações na ultraestrutura e inviabilizando a atividade celular. Para Bharali et al. (2013) [45], a inserção das caudas de acila mais curtas do ramnolipídio na membrana celular pode causar ruptura entre os elementos do citoesqueleto e a membrana plasmática, permitindo que a membrana se desloque dos constituintes citoplasmáticos. Neste trabalho, não foi possível observar a diferença de sensibilidade entre as bactérias Gram-positivas e Gram-negativas.

O surgimento de novas bactérias patogênicas associado ao rápido desenvolvimento de resistência aos antibióticos disponíveis no mercado tem aumentado a importância da descoberta de novos agentes antimicrobianos [46, 47]. Os ramnolipídios constituem um dos biossurfactantes mais estudados, apresentando resultados promissores como potenciais agentes antimicrobiano [48].

\section{CONCLUSÃO}

Os resultados deste trabalho demonstraram que o surfactante produzido pela linhagem Pseudomonas sp. CCMICS 105 apresentou atividade antimicrobiana frente a todas cepas microbianas estudadas. A prospecção de compostos com atividade antimicrobiana, como os biossurfactantes, poderá contribuir para descoberta de potenciais agentes para combater infecções causadas por microrganismos. Trabalhos futuros devem ser realizados para avaliar o perfil de atividade antimicrobiana com amplo espectro de microrganismos, determinar a estrutura química e citotoxicidade deste tensoativo.

\section{REFERÊNCIAS BIBLIOGRÁFICAS}

1. Xu Q, Nakajima M, Liu Z, Shiina T. Biosurfactants for microbubble preparation and application. Int J Mol Sci. 2011 Jan 17;12(1):462-75. doi: 10.3390/ijms12010462

2. Banat IM, Franzetti A, Gandolfi I, Bestetti G, Martinotti MG, Fracchia L, et al. Microbial biosurfactants production, applicatins and future potential. Appl Microbiol Biotechnol. 2010 Jun;87(2):427-44. doi: 10.1007/s00253-010-2589-0

3. Hassan M, Essam T, Yassin AS, Salama A. Optimization of rhamnolipid production by biodegrading bacterial isolates using Plackett-Burman design. Int J Biol Macromol. 2016 Jan;82:573-9. doi: 10.1016/j.ijbiomac.2015.09.057

4. Banat IM, Satpute SK, Cameotra SS, Patil R, Nyayanit. NV. Cost effective technologies and renewable substrates for biosurfactants production. Front Microbiol. 2014 Dec;5:697. doi: 10.3389/fmicb.2014.00697 
5. Santos SC, Fernandez LG, Rossi-Alva JC, Roque MRA. Evaluation of substrates from renewable resources in biosurfactants production by Pseudomonas strains. Afr J Biotechnol. 2010 Aug;9(35):5704-11. doi: 10.5897/AJB10.201

6. Santos SC, Torquato CA, Santos DA. Solução nutritiva alternativa para produção de biossurfactante. Patente: Privilégio de Inovação. Número do registro: BR10202002115. Depósito: 15/10/2020.F. Brasil: Instituto Nacional da Propriedade Industrial; 2020.

7. Bognolo G. Biosurfactants as emulsifying for hydrocarbons. Physicochem Eng Aspects. 1999 Jul;152(1):41-52. doi: 10.1016/S0927-7757(98)00684-0

8. Saikia RR, Deka S. Biosurfactants: structure, function and their properties. Int J Educ Sci Res Rev. 2015;2:5-20.

9. Sun S, Wang Y, Zang T, Wei J, Wu H, Wei C, et al. A biosurfactant producing Pseudomonas aeruginos S5 isolated from coking wastewater and its application for bioremediation of polycyclic aromatic hydrocarbons. Bioresource Technol. 2019 Jun;281:421-8. doi: 10.1016/j.biortech.2019.02.087

10. Santos SC. Biossurfactantes: potenciais agentes biorremediadores. Cadernos de Prospecção. 2019 Mar;12(5):1531-40. doi: 10.9771/cp.v12i5\%20Especial.33191

11. Ndlovu T, Rautenbach M, Khan S, Khan W. Variants of lipopeptides and glycolipids produced by Bacillus amyloliquefaciens and Pseudomonas aeruginosa cultured in diferent carbon substrates. AMB Expr. 2017 May;109(7):1-13. doi: 10.1186/s13568-017-0367-4.

12. Ohadi M, Forootanfar H, Dehghannoudeh G, Eslaminejad D, Ameri A, Shakibaie M, et al. Antimicrobial, anti-biofilm, and anti-proliferative activities of lipopeptide biosurfactant produced by Acinetobacter junii B6. Microbial Pathogenesis. 2020 Jan;138:103806. doi: 10.1016/j.micpath.2019.103806

13. Ahimou F, Jacques P, Deleu M. Surfactin and iturin A effects on bacillus subtilis surface hydrophobicity. Enzyme Microb Technol. 2000 Dec;27(10):749-54. doi: 10.1016/s01410229(00)00295-7

14. Kim KJ, Yoo DS, Kim YB, Lee BS, Shin DH, Kim K. Characteristics of sophorolipid as an antimicrobial agent. J Microbiol Biotechnol. 2002 Apr;12(2):235-41.

15. Arutchelvi JI, Bhaduri S, Uppara PV, Doble M. Mannosylerythritol lipids: a review. J Ind Microbiol Biotechnol. 2008 Dec;35(12):1559-70. doi: 10.1007/s10295-008-0460-4

16. Araújo JS, Rocha JC, Oliveira Filho MA, Ribeiro VT, Vasconcelos LTCP, Araújo NK, et al. Production of rhamnolipids by Pseudomonas aeruginosa AP029-GLVIIA and application on bioremediation and as a fungicide. Biosci Biotechnol Res Asia. 2020 Sep;17(3):467-77. doi: $10.13005 / \mathrm{bbra} / 2850$

17. Santos SC, Oliveira LKX, Santos SN, Santos NPC, Quintella CM, Almeida PF, et al. Biosurfactant production from Pseudomonas strains isolated in rhizospheric soils from semi-arid area of Bahia, Brazil. Sitientibus, Sér Ciênc Biol. 2012 Jun;12(1):157-63. doi: 10.13102/scb121

18. Castro DCM, Campos ACV, Silva SC, Quintella CM, Santos SC. Tolerância a metais pesados e produção de surfactante por Pseudomonas fluorescens Slim15. Sci Plena. 2020 Oct;16(10):106201. doi: 10.14808/sci.plena.2020.106201

19. Bodour AA, Miller-Maier RM. Application of a modified drop-collapse technique for surfactant quantitation and screening of biosurfactant-producing microorganisms. J Microbiol Meth. 1998 May;32(3):273-80. doi: 10.1016/S0167-7012(98)00031-1

20. Das M, Das SK, Mukherjee RK. Surface active properties of the culture filtrates of a Micrococcus species grown on n-alkenes and sugars. Bioresour Technol. 1998 Mar 1;63(3):231-5. doi: 10.1016/S0960-8524(97)00133-8

21. Lotfabad TB, Shourian M, Roostaazad R, Najafabadi AR, Adelzadeh MR, Noghabi KA. An efficient biosurfactant-producing bacterium Pseudomonas aeruginosa MR01, isolated from oil excavation areas in south of Iran. Colloids Surf. B Biointerfaces. 2009 Mar 1;69(2):183-93. doi: 10.1016/j.colsurfb.2008.11.018

22. National Committee for Clinical Laboratory Standards (NCCLS). Methods for dilution antimicrobial susceptibility tests for bacteria that grow aerobically: approved standard. 6. ed. Pennsylvania: NCCLS; 2003.

23. Mohr LC, Capelezzo AP, Rippel T, Ternus RZ, Dalcanton F, Fiori MA, et al. Efeito antimicrobiano de nanopartículas de $\mathrm{ZnO}$ e $\mathrm{TiO}_{2}$ frente as bactérias $S$. aureus e E. coli. Rev CSBEA. 2017;3(1):1-10. doi: $10.5965 / 24473650312017011$

24. Banat IM. Biosurfactants production and possible uses in microbial enhanced oil recovery and oil pollution remediation: a review. Bioresour Technol. 1995 51(1):1-12. doi: 10.1016/09608524(94)00101-6 
25. Gomaa EZ. Antimicrobial activity of a biosurfactant produced by Bacillus licheniformis strain M104 grown on whey. Braz Arch Biol Technol. 2013 Apr;56(2):259-68. doi: 10.1590/S151689132013000200011

26. Oluwaseun AC, Kola OJ, Mishra P, Singh JR, Singh AK, Cameotra SS, et al. Characterization and optimization of a rhamnolipid from Pseudomonas aeruginosa C1501 with novel biosurfactant activities. Sustain Chem Pharm. 2017 Dec;6:26-36. doi: 10.1016/j.scp.2017.07.001

27. Randhawa KKS, Rahman PKSM. Rhamnolipid biosurfactants-past, present, and future scenario of global market. Front Microbiol. 2014 Sep;5:1-7. doi: 10.3389/fmicb.2014.00454

28. Gunther NW, Nunez A, Fett W, Solaiman DK. Production of rhamnolipids by Pseudomonas chlororaphis, a nonpathogenic bacterium. Appl Environ Microbiol. 2005 May;71(5):2288-93. doi: 10.1128/AEM.71.5.2288-2293.2005

29. Mulligan CN. Environmental applications for biosurfactants. Environ Pollut. 2005 Jan;133(2):183-98. doi: 10.1016/j.envpol.2004.06.009

30. Wei Y, Chou C, Chang J. Rhamnolipid production by indigenous Pseudomonas aeruginosa originating from petrochemical wastewater. Biochem Eng J. 2005 Dec 15;27(2):146-54. doi: 10.1016/j.bej.2005.08.028

31. Das P, Yang XP, Ma LZ. Analysis of biosurfactants from industrially viable Pseudomonas strain isolated from crude oil suggests how rhamnolipids congeners affect emulsification property and antimicrobial activity. Front Microbiol. 2014 Dec 22;5:696. doi: 10.3389/fmicb.2014.00696

32. Ebrahimipour G, Gilavand F, Karkhane M, Kavyanifard A, Teymouri M, Marzban A. Bioemulsification activity assessment of an indigenous strain of halotolerant Planococcus and partial characterization of produced biosurfactants. Int J Environ Sci Technol. 2014 Jul;11(5):1379-86. doi: 10.1007/s13762-014-0548-5

33. Harshada K. Biosurfactant: A potent antimicrobial agent. J Microbiol Exp. 2014 Dec;1(5):173-7. doi: 10.15406/jmen.2014.01.00031

34. Nitschke M, Pastore GM. Biossurfactantes: propriedades e aplicações. Quím Nova. 2002 Set;25(5):772-6. doi: 10.1590/S0100-40422002000500013

35. Muller MM, Hormann B, Kugel M, Syldatk C, Hausmann R. Evaluation of rhamnolipid production capacity of Pseudomonas aeruginosa PAO1 in comparison to the rhamnolipid over-producer strains DSM 7108 and DSM 2874. Appl Microbiol Biotechnol. 2011 Feb;89(3):585-92. doi: 10.1007/s00253010-2901-z

36. Carvalho CR, Maia MQ, Sobral M, Pereira GMD, Silva K, Vital MJS, et al. Diversity and antimicrobial activity of culturable endophytic fungi associated with the neotropical ethnomedicinal plants Copaifera langsdorffii and Copaifera pubiflora. South Afr J Bot. 2021 Nov;142:305-15. doi: 10.1016/j.sajb.2021.06.021

37. Hamedi J, Kafshnouchi M, Ranjbaran M. A study on actinobacterial diversity of Hampoeil cave and screening of their biological activities. J Biol Sci. 2019 Nov;26(7):1587-95. doi: 10.1016/j.sjbs.2018.10.010

38. Ghosh S, Kam G, Nijjer M, Stenner C, Cheeptham N. Culture dependent analysis of bacterial diversity in Canada's Raspberry Rising Cave revealed antimicrobial properties. Int J Speleol. 2020 Jan;49(1):43-53. doi: 10.5038/1827-806X.49.1.2291

39. Balan SS, Kumar CG, Jayalakshmi S. Aneurinifactin, a new lipopeptide biosurfactant produced by a marine Aneurinibacillus aneurinilyticus SBP-11 isolated from Gulf of Mannar: purification, characterization and its biological evaluation. Microbiol Res. 2017 Jan;194:1-9. doi: 10.1016/j.micres.2016.10.005

40. Vecino X, Rodríguez-López L, Ferreira D, Cruz JM, Moldes AB, Rodrigues LR. Bioactivity of glycolipopeptide cell-bound biosurfactants against skin pathogens. Int J Biol Macromol. 2018 Apr 1;109:971-9. doi: 10.1016/j.ijbiomac.2017.11.088

41. Gudiña EJ, Rocha V, Teixeira JÁ, Rodrigues LR. Antimicrobial and antiadhesive properties of a biosurfactant isolated from Lactobacillus paracasei ssp. paracasei A20. Lett Appl Microbiol. 2010 Apr;50(4):419-24. doi: 10.1111/j.1472-765X.2010.02818.x

42. El-Sheshtawy ES, Doheim MM. Selection of Pseudomonas aeruginosa for biosurfactant production and studies of its antimicrobial activity. Egypt J Pet. 2014 Mar;23(1):1-6. doi: 10.1016/j.ejpe.2014.02.001

43. Vijayakumar S, Saravanan V. In vitro cytotoxicity and antimicrobial activity of biosurfactant produced by Pseudomonas aeruginosa strain PB3A. Asian J Sci Res. 2015 8(4):510-8. doi: 10.3923/ajsr.2015.510.518

44. Bharali P, Konwar, BK. Production and physico-chemical characterization of a biosurfactant produced by Pseudomonas aeruginosa OBP1 isolated from petroleum sludge. Appl Biochem Biotechnol. 2011 Apr;164:1444-60. doi: 10.1007/s12010-011-9225-Z 
45. Bharali P, Saikia JP, Ray A. Rhamnolipid (RL) from Pseudomonas aeruginosa OBP1: a novel chemotaxis and antibacterial agent. Colloids Surf B Biointerfaces. 2013 Mar;103:502-9. doi: 10.1016/j.colsurfb.2012.10.064

46. Bhullar K, Waglechner N, Pawlowski A, Koteva K, Banks ED, Johnston MD, et al. Antibiotic resistance is prevalent in an isolated cave microbiome. PLoS ONE. 2012 Apr;7:e34953. doi: 10.1371/journal.pone.0034953

47. Monciardini P, Iorio M, Maffioli S, Sosio M, Donadio S. Discovering new bioactive molecules from microbial sources. Microb Biotechnol. 2014 May;7(3):209-20. doi: 10.1111/1751-7915.12123

48. Mandal SM, Barbosa AE, Franco OL. Lipopeptides in microbial infection control: scope and reality for industry. Biotechnol Adv. 2013 Apr;31(2):338-45. doi: 10.1016/j.biotechadv.2013.01.004 